\title{
SABCS 2017: Was gibt es Neues beim Mammakarzinom?
}

\section{Neue therapeutische Strategien beim Mammakarzinom wurden auf dem San Antonio Breast Cancer Symposium (SABCS) in San Antonio/USA, der Fachwelt vorgestellt. Das ONKO-Internetportal berichtet in Video-Interviews, Kon- gressberichten und einer Expertenrunde von den High- lights und den Kontroversen der Konferenz.}

Zu den am stärksten beachteten Studien vom SABCS 2017 gehört die MONALEESA-7-Studie. In der Studie wurde untersucht, ob der CDK4/6-Inhibitor Ribociclib zusammen mit einer Hormontherapie und dem GnRH-Analogon Goserelin das progressionsfreie Überleben auch bei prä- und perimenopausalen Patientinnen verbessert. Denn bisher lagen keine Daten für den Einsatz der CDK-Inhibitoren für die Erstlinientherapie bei prä- und perimenopausalen Patientinnen vor. Es zeigte sich, dass der CDK4/6Hemmer auch für junge Patientinnen einen therapeutischen Vorteil bringt.

Interessante Daten gab es beim SABCS 2017 auch zur erweiterten Hormontherapie. Erweiterte Hormontherapien haben sich grundsätzlich als vorteilhaft für Patientinnen mit hormonempfindlichem Mammakarzinom herausgestellt, die auch nach adjuvanter Therapie mit Tamoxifen oder einem Aromatasehemmer weiterhin ein Rezidivrisiko haben. In der Phase-III-Studie ABCSG-16 wurde die optimale Dauer der erweiterten Therapie mit Anastrozol nach initialer Tamoxifen- bzw. Aromatasehemmer-Behandlung oder Sequenz untersucht. Im Ergebnis verbes-

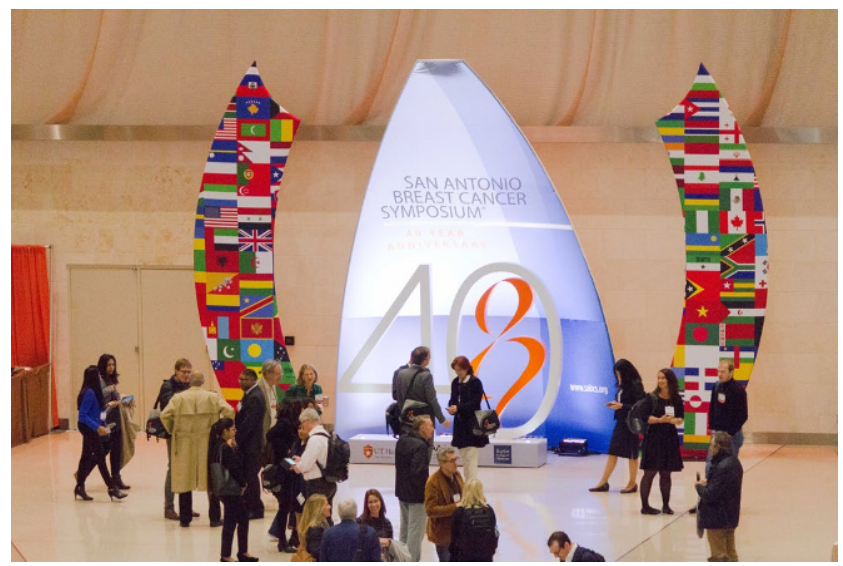

Abb. 1 - Impressionen vom San Antonio Breast Cancer Symposium (৫ dkg-web.gmbh) sert eine erweiterte Therapie mit fünf Jahren Anastrozol im Vergleich zu zwei Jahren im Anschluss an eine endokrine Therapie von vier bis sechs Jahren nicht das krankheitsfreie Überleben. Das Ergebnis spricht also gegen eine Langezeitbehandlung.

Für Gesprächsstoff sorgten beim SABCS ebenfalls Daten aus der Phase-III-Studie GeparSepto, die einen klinischen Nutzen von nab-Paclitaxel gegenüber Lösungsmittel-basiertem Paclitaxel bei der Behandlung der Patientin mit einem primären Mammakarzinom nachweisen konnte: Es zeigte sich beim Vergleich beider Substanzen nach wöchentlicher Gabe gefolgt von Anthrazyklin/ Cyclophosphamid im Rahmen einer neoadjuvanten Chemotherapie bei primärem Brustkrebs ein verbessertes krankheitsfreies Überleben unter nab-Paclitaxel.

Mehr zur aktuellen Bandbreite der Therapieoptionen beim Mammakarzinom erfahren Sie auch in der Aufzeichnung unserer Expertenrunde vom SABCS 2017 mit den renommierten Mammakarzinom-Experten Prof. Christian Jackisch (Offenbach), Prof. Sibylle Loibl (Frankfurt), Prof. Christoph Thomssen (Halle), Prof. Cornelia Liedtke (Lübeck), Prof. Volkmar Müller (Hamburg), Prof. Michael Untch (Berlin), Dr. Rachel Würstlein (München), Prof. Wolfgang Janni (Ulm) und Prof. Tanja Fehm (Düsseldorf).

Alle Videos und Berichte können Sie online aufrufen oder herunterladen unter www.krebsgesellschaft.de/sabcs2017 oder unter diesem QR-Code:

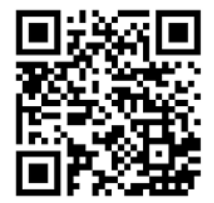

\section{Korrespondenzadresse}

\section{Pia Nitz}

Redaktion ONKO-Internetportal

In Kooperation mit der Deutschen Krebsgesellschaft e.V.

Tel. $030-810316112$

pia.nitz@dkg-web.de 